\title{
BMJ Open Quality Implementation of vaginal preparation prior to caesarean section
}

\author{
Adam D Jakes, ${ }^{1}$ Annie Bell, ${ }^{1}$ Lilian Chiwera, ${ }^{2}$ Jilly Lloyd ${ }^{1}$
}

To cite: Jakes $A D$, Bell A, Chiwera L, et al. Implementation of vaginal preparation prior to caesarean section. BMJ Open Quality 2020;9:e000976. doi:10.1136/ bmjoq-2020-000976

- Additional material is published online only. To view please visit the journal online (http://dx.doi.org/10.1136/ bmjoq-2020-000976).

Received 23 March 2020

Revised 25 June 2020 Accepted 9 July 2020
Check for updates

(c) Author(s) (or their employer(s)) 2020. Re-use permitted under CC BY-NC. No commercial re-use. See rights and permissions. Published by BMJ.

${ }^{1}$ Women's Services, Guy's and Saint Thomas' NHS Foundation Trust, London, UK

${ }^{2}$ Infection Control, Guy's and Saint Thomas' NHS Foundation Trust, London, UK

Correspondence to

Dr Adam D Jakes;

a.jakes@nhs.net

\begin{abstract}
Introduction Surgical site infections following caesarean section are associated with significant morbidity. Vaginal preparation is the cleansing of the vaginal epithelium with an antibacterial solution to reduce the bacterial load and therefore reduce ascending genital tract infection. It is recommended by the WHO and a Cochrane review in 2018 concluded that vaginal preparation immediately before caesarean section probably reduces the rates of postoperative endometritis.

Objective To implement vaginal preparation prior to caesarean section at Guy's and St Thomas' Hospital NHS Foundation Trust and reduce rates of deep surgical site infections.

Methods The protocol (included within the appendices) for vaginal preparation prior to caesarean section was developed after reviewing the available evidence. Two vaginal preparation champions, a midwife and a scrub nurse, were selected to help promote and assist in the implementation. The first implementation cycle included elective and category II and III caesarean sections. To ensure acceptability, 20 women were asked to complete a questionnaire following vaginal preparation. Once the intervention was being performed in $>85 \%$ of eligible women, the inclusion criteria was expanded to include category I caesarean sections.
\end{abstract}

Results Twelve months following implementation, vaginal preparation was still being performed in $89 \%$ of eligible women. The deep surgical site infection rate is now the lowest recorded in the last 6 years. Vaginal preparation prior to caesarean section was acceptable to pregnant women and no adverse effects were reported.

Conclusions Vaginal preparation prior to caesarean section has been successfully implemented at Guy's and St Thomas' Hospital NHS Foundation Trust. This simple, cheap intervention, performed with readily available materials, is still being performed in a high number of caesarean sections 12 months post-implementation. It has resulted in a reduction in deep surgical site infections. Involvement of key stakeholders and the recruitment of vaginal preparation champions were key to success.

\section{PROBLEM}

A surgical site infection (SSI) is defined as an infection that occurs within 30 days after a surgical procedure. ${ }^{1}$ It can be divided into superficial incisional, deep incisional and organ/space infections. SSIs are considered superficial when involving only the skin and subcutaneous tissue, for example, cellulitis or a superficial wound infection. Deep incisional
SSIs involve discharging wounds with deep tissue involvement of the fascial and muscle layers. Organ/space SSIs extend beyond the fascial and muscle layers, for example, an abscess or collection. Deep incisional and organ/space SSIs following a caesarean section are commonly grouped together to include postoperative endometritis and intraabdominal collections. ${ }^{1}$

SSIs are associated with a significant morbidly and mortality. High SSI rates inevitably result in poor patient outcomes and significant financial burden to healthcare organisations. Rates vary nationally between hospitals and regions because of variations in surgical care and different data collection approaches used to record SSIs at hospital and community level.

Guy's and St Thomas' Hospital NHS Foundation Trust is a large, tertiary hospital in central London. The maternity service delivers roughly 6800 babies each year, with a caesarean section rate of $35 \% .^{2}$ The trust implemented SSI surveillance for both elective and emergency caesarean sections in 2010 in line with Public Health England recommendations. ${ }^{3}$ This consists of surveillance forms, postdischarge questionnaires and telephone interviews. Post-discharge response rates using postal questionnaires and telephone surveys for the validated quarter in 2019 were $91.5 \% .^{4}$ Superficial SSIs have been significantly reduced by the implementation of standardised skin preparation, changing the timing of prophylactic antibiotic administration from post-cord clamping to pre-surgical incision, the use of evidence-based suture material and negative pressure dressings for high-risk women. ${ }^{4}$ Unfortunately, deep surgical site (and organ/space) infections such as endometritis have remained constant over the past 5 years. There were seven deep SSIs in 2017 and five in 2018.

The aim of the quality improvement project was to implement vaginal preparation prior to caesarean section and reduce rates of deep SSIs. 


\section{BACKGROUND}

A caesarean section is the most common obstetric operation and rates are increasing worldwide ${ }^{56}$ The incidence of SSI following caesarean section varies but has been reported in up to $10 \%$ of women. ${ }^{6}$ Obesity appears to be the biggest risk factor for developing an SSI. ${ }^{6}$ Consequences of caesarean section SSIs include chronic pelvic pain, delay in returning to normal activities, depression, longer hospital stay and increased hospital costs. ${ }^{7}$

Superficial SSIs are much more common than deep or organ/space SSI following caesarean section. At Guy's and St Thomas' Hospital NHS Foundation, superficial SSIs have been significantly reduced through a number of improvement strategies, but deep surgical SSI remained constant.

Vaginal preparation is the cleansing of the vaginal epithelium with an antibacterial solution to reduce the bacterial load and therefore reduce ascending genital tract infection. A Cochrane review in 2018 concluded that vaginal preparation with povidone-iodine or chlorhexidine solution immediately before caesarean delivery probably reduces the rates of postoperative endometritis. ${ }^{8}$ It was also recommended in a systematic review and meta-analysis of best practice perioperative strategies for preventing caesarean section SSIs published in the $B J O G{ }^{7}$ Vaginal preparation with povidone-iodine immediately before caesarean section is recommended by the WHO. ${ }^{9}$ Women undergoing caesarean delivery who were already in labour or who had ruptured membranes benefited the most from this intervention. ${ }^{8}$

There is no agreed protocol in the literature for vaginal preparation prior to caesarean section.

A decision was made to implement vaginal preparation prior to caesarean section at Guy's and St Thomas' Hospital NHS Foundation Trust in an attempt to reduce rates of deep SSIs.

\section{MEASUREMENT}

All caesarean sections performed in theatre are recorded in a register by the scrub team. This was adapted to also record whether a woman has had vaginal preparation prior to caesarean section in eligible women.

Rates of SSI are routinely collected by the trust Infection Control Surveillance Team. This consisted of surveillance forms, post-discharge questionnaires and telephone interviews. Post-discharge questionnaires were posted to all women at day 25 post-caesarean section to arrive in time for the 30-day follow-up. All women were telephoned from day 30 post-caesarean section, with up to three attempts to make contact. Women were asked about any history of SSI symptoms using a standardised template and whether they had received antibiotics. No additional data collection was required for the quality improvement project.

No reduction in superficial SSIs was expected following the implementation of vaginal preparation. Due to the very small number of deep SSIs each year, it was possible that there may not be a significant drop in the number of infections following the intervention. However, the infection rates were not expected to rise, and vaginal preparation is seen as a best practice perioperative strategy for preventing caesarean section SSIs.

To ensure the intervention was acceptable to women, a patient questionnaire was designed to inquire about their experience following vaginal preparation. Twenty women were approached the following day after their caesarean section and asked to complete a questionnaire.

\section{DESIGN}

\section{Scoping}

The project team was made up of an obstetric registrar, obstetric consultant and labour ward lead, the Infection Control Surveillance Team Leader and obstetric theatre midwifery practice lead. Early feedback during scoping of the project revealed that the phrase 'vaginal cleansing', which is used by the WHO and Cochrane review, may not be appropriate. There was concern that it implied that the vagina was dirty, and therefore the phrase 'vaginal preparation' was agreed and used throughout the project.

A survey was sent to the labour ward leads within the UK via the British Intrapartum Society mailing list, labour ward lead forums and contacting individual centres in December 2018. Each labour ward lead was asked whether they had successfully implemented vaginal preparation prior to caesarean section within their unit. Data were also collected regarding the barriers to implementation. Responses were collected as either fully implemented, in the process of implementation, ongoing consultation regarding implementation or no current plans for implementation. In those who had successfully implemented vaginal preparation, data were collected regarding the date of implementation, the category of caesarean section included and the cleansing solution and technique used.

Fifty-two maternity units responded. Five units have successfully implemented vaginal preparation prior to caesarean section. All units used $10 \%$ povidone-iodine solution and had implemented vaginal preparation within the last year. Only one unit offered it to women undergoing an elective caesarean section while three units offered it to category I caesarean sections. Four out of five units included caesarean section categories II and III. Four of these units were in London. Of the units who had not implemented vaginal preparation, 3 units were in the process of implementing and 21 were considering implementing the process. Twenty-three units had no plans to implement. The common themes regarding barriers to implementation were resistance from staff, difficulty changing culture and concerns from neonatal teams regarding solution used.

\section{Protocol development}

The vaginal preparation prior to caesarean section protocol was developed after reviewing the available evidence. ${ }^{7-9}$ The protocol was presented and approved by 


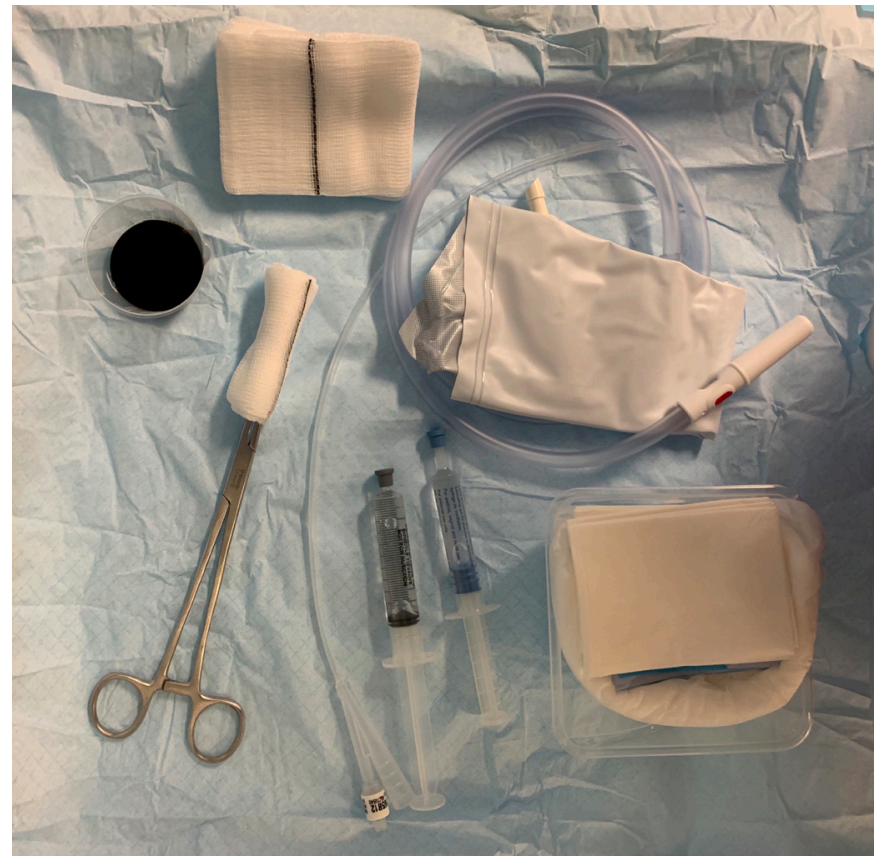

Figure 1 Equipment set-up in theatre.

the SSI committee, the Women's Services Clinical Governance and the Trust Risk and Assurance Committee. The decision to use $10 \%$ povidone-iodine solution was made due to the obstetric theatre staff's familiarity with its use for vulvovaginal cleaning in obstetric anal sphincter injury repair. It was also being used in other units across the region and had been used in the highest number of vaginal preparation clinical trials.

Concerns raised by stakeholders included the theoretical effects of the iodine on the neonatal thyroid function, discolouration of the baby's scalp and postpartum vaginal irritation. A review of the literature demonstrated no evidence that neonatal thyroid function was adversely affected by iodine skin application. There is only a short duration of contact with the baby's scalp and midwives were told to simply wipe away any excess iodine seen at birth. We agreed to perform a questionnaire in 20 women following vaginal preparation to determine if vaginal irritation was an issue.

Women were excluded if they declined vaginal preparation after explanation of the process. In addition, face presentation and failed instrumental delivery were also exclusion criteria. If an allergy to povidone-iodine solution or shellfish was documented, chlorhexidine $2 \%$ aqueous solution was used instead. Only NHS patients were included, consultants performing private caesarean sections were given the choice whether they wanted to perform vaginal preparation on their patients.

A plan to start with women undergoing an elective or emergency caesarean section (category II or III) was agreed. Category I are the most time-critical emergency caesarean sections which involve an immediate threat to life of the woman or fetus. A standard of 30 minutes from decision to operate to the delivery of the fetus is recommended. ${ }^{10}$ In comparison, National Institute for Health and Care Excellence recommends performing a category 2 caesarean section, in most situations, within $75 \mathrm{~min}$ of making the decision. A category III caesarean section is performed when there is no maternal or fetal compromise, but early delivery is required. Due to the urgency of category I caesarean sections, these women were excluded from the first change cycle until staff became familiar with the process with minimal delays.

Only verbal consent was required for vaginal preparation. It was gained by the healthcare professional at the same time as consent for vaginal examination or urinary catheterisation.

The healthcare professional cleaned the vagina prior to emergency caesarean section. This was performed following urinary catheterisation or at the 'time out' if a catheter was in situ. If a vaginal examination was required to make a decision regarding method of delivery, vaginal preparation was to be performed afterwards. Sponge holders with a single gauze swab were used from the scrub set, with the gauze included in the final swab count for that theatre case. Figure 1 demonstrates the equipment set up in theatre.

The full vaginal preparation prior to caesarean section protocol can be found in online supplementary appendix 1.

Two vaginal preparation champions, a midwife and a scrub nurse, were selected to help promote and assist in the implementation.

\section{STRATEGY}

\section{Change cycle 1}

To start, a bulletin announcing the implementation of vaginal preparation prior to caesarean section was placed in the Women's Services weekly newsletter and a global email circulated to the maternity services. Vaginal preparation champions were recruited and trained (one

\section{Box 1 Questionnaire responses}

1. Do you remember staff discussing with you that they will be cleaning your vagina before the caesarean section?

- Yes-15/20 (75\%)

- No-5/20 (25\%)

2. Have you experienced any abnormal vaginal discharge following the caesarean section?

- Yes-0 (0\%)

- No-20/20 (100\%)

3. Are you experiencing any vaginal pain or irritation?

- Yes-0 $(0 \%)$

- No-20/20 (100\%)

4. Did you noticed any discolouration of the babies scalp immediately following the caesarean section (not jaundice)?

- Yes-0 $(0 \%)$

- No-20/20 (100\%)

5. Did the staff ensure they did their best to maintain your dignity throughout the caesarean section?

- Yes-20/20 (100\%)

- No-0 $(0 \%)$ 


\begin{tabular}{llllrrrl}
\multicolumn{2}{l}{ Table 1 Results following implementation } & & & & & \\
\hline \multicolumn{2}{l}{} & & Category I & Category II & Category III & Elective & Total \\
\hline Change cycle 1 & & & & & & \\
Vaginal preparation & Yes & 0 & 68 & 33 & 53 & 154 \\
& No & 0 & 16 & 1 & 6 & 23 \\
Total & & 0 & 84 & 34 & 59 & 177 \\
Change cycle 2 & & & & & & \\
Vaginal preparation & Yes & 6 & 51 & 33 & 79 & 169 \\
& No & 7 & 9 & 3 & 2 & 21 \\
Total & & 13 & 60 & 36 & 81 & 190
\end{tabular}

No vaginal preparation was performed prior to implementation.

midwife and one scrub nurse). One week prior to the implementation of vaginal preparation, the practice development midwives arranged drop-in sessions for doctors and midwives to learn how to perform vaginal preparation. This was done using a pelvic mannequin and staff were 'signed off' as competent to perform the procedure. The vaginal preparation prior to caesarean section protocol was printed, laminated and placed in easy view within the obstetric theatres.

Vaginal preparation was implemented for all elective and category II and III caesarean sections in January 2019. During the first 2 weeks of implementation, vaginal preparation champions assisted in the encouragement and support of staff to perform the intervention.

Twenty women at random were asked to complete a questionnaire about their experience 1 day following vaginal preparation. No women declined to answer the questionnaire.

Six months post-implementation, all caesarean sections within a 1-month period were reviewed to assess whether vaginal preparation was being performed in all eligible women.

\section{Change cycle 2}

Due to the success of the implementation, the decision to include category I caesarean sections was agreed 6 months post-implementation (July 2019).
Another Women's Servicers weekly' bulletin and global email to maternity services was circulated informing staff of the change.

In addition, the caesarean section data entry form on the electronic maternity record (BadgerNet maternity, Clevermed) was amended to include a mandatory tickbox to confirm vaginal preparation had been performed. This ensured that all vaginal preparation was electronically recorded making future audit more efficient and less time consuming. In the event of any future post-caesarean section endometritis, the risk team can review whether vaginal preparation was performed.

Twelve months post-implementation, all caesarean sections within a 1-month period were reviewed to assess whether vaginal preparation was being performed in all eligible women and was a sustainable intervention.

\section{RESULTS}

No adverse events have been reported since the implementation of vaginal preparation. No neonatal concerns have been raised by the women or the paediatric doctors.

\section{Acceptability}

Twenty women were asked to complete a questionnaire 1 day following vaginal preparation. The questions and responses are listed in box 1 . No women complained of

Table 2 Surgical site infection rates

\begin{tabular}{lllllll}
\hline & $\begin{array}{l}\text { Post-discharge } \\
\text { diagnosis }\end{array}$ & $\begin{array}{l}\text { Inpatient/ } \\
\text { Readmission }\end{array}$ & $\begin{array}{l}\text { Emergency } \\
\text { caesarean }\end{array}$ & $\begin{array}{l}\text { Elective } \\
\text { caesarean }\end{array}$ & $\begin{array}{l}\text { Superficial } \\
\text { infection }\end{array}$ & $\begin{array}{l}\text { Deep and } \\
\text { organ/space }\end{array}$ \\
Total
\end{tabular}

*Post-discharge surveillance telephone surveys could not be completed for this year due to staff shortage. 
abnormal or discoloured vaginal discharge which was an initial concern from stakeholders. This is likely due to the cleaning of the vagina post-caesarean section to remove clots and ensure there is no ongoing bleeding from the uterus. No woman complained of vaginal irritation or pain following vaginal preparation. There were also no concerns about discolouration of the baby scalp.

During the two cycles of data collection, only one woman declined vaginal preparation after being appropriately counselled. It is understood that this is a rare event.

\section{VAGINAL PREPARATION}

Six months following implementation, the uptake of vaginal preparation prior to caesarean section was reviewed over 1 month (table 1). After excluding the category I caesarean sections and failed instrumental deliveries, there were 177 eligible women. One hundred and fifty-four $(87 \%)$ had vaginal preparation performed; category $\mathrm{II}=68(81 \%)$, category $\mathrm{III}=33(97 \%)$ and elective caesarean section $53(90 \%)$.

Twelve months following implementation, the uptake of vaginal preparation prior to caesarean section was reviewed over 1 month (table 1). After excluding the failed instrumental deliveries, there were 190 eligible women (vaginal preparation data were missing for six women who were also excluded). One hundred and sixtynine $(89 \%)$ had vaginal preparation performed; category $\mathrm{I}=6(46 \%)$, category $\mathrm{II}=51(85 \%)$, category $\mathrm{III}=33(92 \%)$ and elective caesarean section $79(98 \%)$. Of the eligible women who did not received vaginal preparation, three were a caesarean section under general anaesthetic, one was a cord prolapse, one declined the intervention, one was allergic to iodine and the remaining women had no specific reason why it was not performed.

\section{Surgical site infections}

Prior to the implementation of vaginal preparation, there were five deep or organ/space infections between January and December 2018. Following implemented in January 2019, there have been no deep or organ/space infections (table 2). This can be extrapolated to suggest cost savings for the trust, reduced unnecessary admissions and antibiotic prescriptions.

\section{LESSONS AND LIMITATIONS}

The project aim was to implement vaginal preparation prior to caesarean section at Guy's and St Thomas' Hospital NHS Foundation Trust. The intervention has been successfully implemented and sustained for over 12 months. Involvement of key stakeholders and the recruitment of vaginal preparation champions were key to success. Vaginal preparation prior to caesarean section is acceptable to pregnant women and no adverse effects were reported. This intervention is simple, cheap and performed with readily available materials.
The decision to stagger the inclusion of category I caesarean sections until the process had become establish worked very well. This ensured that staff did not struggle with a new intervention and the time-sensitive nature of a category I caesarean section. Once the intervention was being performed in $>85 \%$ of eligible women, the inclusion criteria was expanded with the support of the clinical governance team.

The deep SSI rate is now the lowest recorded in the last 6 years. No statistical analysis was possible due to the relatively small numbers of women who have had the intervention and the short timescale observed. In addition, the number of deep SSIs even before the intervention was very low. SSI surveillance will continue.

Future work will focus on considering its use in failed instrumental deliveries. The process and cleansing solution will be reviewed annually to ensure it is consistent with current evidence.

\section{CONCLUSION}

Vaginal preparation prior to caesarean section has been successfully implemented at Guy's and St Thomas' Hospital NHS Foundation Trust. This simple, cheap intervention, performed with readily available materials, is still being performed in a high number of caesarean sections 12 months post-implementation. It has resulted in a reduction in deep SSIs. Involvement of key stakeholders and the recruitment of vaginal preparation champions were key to success. A protocol for vaginal preparation prior to caesarean section was developed and will be useful to any obstetric department interested in implementing this simple, cheap intervention.

Acknowledgements The authors thank the obstetric theatre staff for all their help and hard work with implementing vaginal preparation prior to caesarean section.

Contributors ADJ led the proposal, implementation and evaluation of the project. $A B$ supported and supervised the theatre team during implementation, assisted in staff education and sourced materials. LC collated and reported the SSI data. $\mathrm{JL}$ supervised the project as labour ward lead and contributed to stakeholder engagement through directorate senior management meetings. All authors contributed to the development of the vaginal preparation protocol. All authors reviewed and approved the final version of the article.

Funding The authors have not declared a specific grant for this research from any funding agency in the public, commercial or not-for-profit sectors.

Competing interests None declared.

Patient and public involvement Patients and/or the public were not involved in the design, conduct, reporting or dissemination plans of this research.

Patient consent for publication Not required.

Provenance and peer review Not commissioned; externally peer reviewed.

Data availability statement All data relevant to the study are included in the article or uploaded as supplementary information.

Open access This is an open access article distributed in accordance with the Creative Commons Attribution Non Commercial (CC BY-NC 4.0) license, which permits others to distribute, remix, adapt, build upon this work noncommercially, and license their derivative works on different terms, provided the original work is properly cited, appropriate credit is given, any changes made indicated, and the use is non-commercial. See: http://creativecommons.org/ licenses/by-nc/4.0/. 


\section{REFERENCES}

1 Public Health England (2013). Protocol for the surveillance of surgical site infection. version 6. Available: https://www.gov.uk/ government/publications/surgical-site-infection-surveillanceservice-protocol-procedure-codes-and-user-manual [Accessed Jun 2020].

2 Guy's \& St Thomas' Hospital NHS Foundation Trust. January. Caesarean section surgical site infection surveillance report. infection prevention and control, 2020.

3 Public Health England. Protocol for the surveillance of surgical site infection. version 6 June 2013. public health England, 2013. Available: https://assets.publishing.service.gov.uk/government/ uploads/system/uploads/attachment_data/file/633775/surgical_site_ infections_protocol_version_6.pdf [Accessed Jun 2020].

4 Guy's \& St Thomas' Hospital NHS Foundation Trust. Women's services birth data 2019.

5 Boerma T, Ronsmans C, Melesse DY, et al. Global epidemiology of use of and disparities in caesarean sections. Lancet 2018;392:1341-8.
6 Wloch C, Wilson J, Lamagni T, et al. Risk factors for surgical site infection following caesarean section in England: results from a multicentre cohort study. BJOG 2012;119:1324-33.

7 Martin EK, Beckmann MM, Barnsbee LN, et al. Best practice perioperative strategies and surgical techniques for preventing caesarean section surgical site infections: a systematic review of reviews and meta-analyses. BJOG 2018;125:956-64.

8 Haas DM, Morgan S, Contreras K, et al. Vaginal preparation with antiseptic solution before cesarean section for preventing postoperative infections. Cochrane Database Syst Rev 2018;30:CD007892.

9 WHO. Who recommendation on vaginal cleansing with povidoneiodine immediately before caesarean section, 2020. Available: https://extranet.who.int/rhl/topics/preconception-pregnancychildbirth-and-postpartum-care/who-recommendation-vaginalcleansing-povidone-iodine-immediately-caesarean-section [Accessed Jun 2020].

10 Royal College of Obstetricians \& Gynaecologists, The Royal College of Anaesthetists. Classification of urgency of caesarean section - $A$ continuum of risk. Good Practice No. 11, 2010. 